\title{
DENKMÄLER
}

$$
\text { DES }
$$

\section{KLASSISCHEN ALTERTUMS.}





\title{
DENKM ÄLER
}

nus

\section{KLASSISCHEN ALTERTUMS}

TUR ERLÄUTERUNG DES LEBENS

DER

\section{GR I E CHE N UND R Ö M E R}

IN

\author{
REIIGION, KUNST' UND STT'TE.
}

\author{
LEXIKALISCH BEARBEITET \\ YON
}

B. ARNOLD, E. ASSMANN, H. BLÜHNER, R. BORRMANN, W. DEECKE, E. FABRICIUS, A. FLASCH, P. GRAE, A. HOLM, K. VON JAN, L. JULUUS, G. KA WERAU, J. MATZ, A. MILCHHÖFER, A. MÜLIER, O. RICHTER, H. VON ROHDEN, L. VON SYBEL. A. TRENDELENBURG, C. WAIDSTEIN, R. WELL, E. WÖLFFLIN UND DEM HERAUSGEBER

A. BAUMEISTER.

111. BAND (RECHENBRETT-ZWÖLFGÖTTER). MIT 82 ABBILDUNGEN, \& KARTEN IND XLII TAFELN.

MÜNCHEN UND IEIPZIG.

DRUCK UND VERLAG VON R. OLDENBOUR(T. 
